\title{
Uso de C3F8 no descolamento da membrana de Descemet pós-facectomia
}

\author{
C3F8 use in Descemet detachmentaftercataract surgery
}

\author{
Abrahão da Rocha Lucena ${ }^{1}$ \\ David da Rocha Lucena ${ }^{2}$ \\ Emília Lucena Macedo ${ }^{3}$ \\ Juliana de Lucena Martins Ferreira ${ }^{4}$ \\ Aristóteles Rolim de Lucena ${ }^{5}$
}

\begin{tabular}{|l|}
\hline RESUMO \\
\hline Introdução: O descolamento da membrana de Descemet é complicação \\
rara, mas devastadora após facectomia. Algumas alternativas têm sido \\
usadas para reposição da Descemet: bolha de ar, sutura com transfixação \\
da córnea, viscoelástico associado com bolha de ar e gás SF6 ou C3F8. O \\
transplante de córnea é o último recurso utilizado. Objetivo: Avaliar os \\
resultados anatômicos e funcionais, do descolamento iatrogênico da \\
membrana de Descemet, com uso de C3F8(16\%) na câmara anterior em seis \\
olhos pós-facectomia. Métodos: Após colocação de 0,5 ml do gás em \\
câmara anterior avaliou-se localização justa-estromal da membrana de \\
Descemet em lâmpada de fenda. Os olhos com deslocamento superior/ \\
central eram orientados a ficar em decúbito elevado por dois dias e no caso \\
do deslocamentoinferior solicitamos decúbito dorsal comleve supraversão. \\
Resultados: A média de idade foi de 71,3 $\pm 9,3$ anos, sendo quatro do sexo \\
feminino e dois do sexo masculino. Todos os olhos tiveram sucesso com \\
a colocação do C3F8, havendo aumento da pressão intra-ocular em um \\
caso. O edema corneano regrediu a partir do segundo dia com resolução \\
completa até o quarto dia. A acuidade visual melhorou em todos os casos \\
logo após regressão do edema, sendo reavaliada quarenta e cinco dias \\
depois. Conclusão: A introdução unicamente do gás C3F8 não expansivo \\
para colar a membrana de Descemeté citado pela primeira vez na literatura \\
brasileira. O restabelecimento da acuidade visual é rápida, o método é \\
seguro, efetivo e de fácil execução, sendo realizado sob anestesia tópica. \\
\hline
\end{tabular}

Descritores: Extração de catarata/efeitos adversos; Membrana de Descemet; Fluorocarbonetos/administração \& dosagem; Edema da córnea/quimioterapia; Injeções

\section{INTRODUÇÃO}

A membrana de Descemet é uma lâmina basal de cerca de $10 \mu \mathrm{m}$ de espessura que reveste a porção posterior do estroma e o separa do endotélio, responsável por sua secreção. Encontra-se frouxamente aderida ao estroma, é homogênea, altamente refrátil, flexível e elástica (apesar de não ter fibras elásticas). Identificam-se duas zonas distintas: anterior, elástica, e a posterior, constituída de lâmina basal. Esta membrana é resistente a infecções e possui uma capacidade parcial de se regenerar, embora muitas vezes de maneira imperfeita ${ }^{(1)}$.

O descolamento da membrana de Descemet não é uma complicação freqüente, mas devastadora após uma cirurgia de catarata. Descolamentos pequenos e periféricos raramente são problemáticos, mas os extensos e/ou próximos ao eixo visual podem afetar em muito a visão dos pacientes, sendo necessário recorrer a transplante penetrante de córnea ${ }^{(2-3)}$. 
Um tratamento tradicional é a conduta expectante, na esperança do recolamento espontâneo. Outras alternativas têm sido usadas na tentativa de reposição da membrana de Descemet: bolha de ar (descolamento pequeno e periférico), sutura com transfixação de toda córnea, viscoelástico associado com bolha de ar, gás (hexafluoreto de enxofre ou SF6 e o perfluorpropano ou C3F8) associado ou não com sutura ${ }^{(3-8)}$.

\section{OBJETIVO}

Avaliar os resultados anatômicos e funcionais, do descolamento iatrogênico da membrana de Descemet, com uso de C3F8 na câmara anterior em seis olhos pós-facectomia.

\section{MÉTODOS}

Realizou-se estudo prospectivo em seis olhos com descolamento da membrana de Descemet pós-facectomia no departamento de córnea e refrativa do Centro Avançado de Retina e Catarata de fevereiro de 2002 até maio de 2004. Dos seis casos de descolamento, cinco foram após facoemulsificação e um por extração extra-capsular do cristalino.

O PAM (potencial de acuidade macular) foi realizado no pré-operatório de todos os pacientes, assim como a acuidade visual (AV) pré-operatória. No pós-operatório, um médico especialista em córnea avaliou: AV, biomicroscopia (observando edema corneano, anatomia: extensão e localização do descolamento). A medida da pressão intra-ocular e o acompanhamento biomicroscópico foi realizado com dois, seis, 45 e 180 dias de pós-operatório.

O descolamento era filmado e fotografado, media-se a pressão intra-ocular e encaminhava o paciente ao bloco cirúrgico para colocação da bolha não expansiva $(16,0 \%)$ do gás C3F8, sob anestesia tópica com cloridrato de oxibuprocaína 0,4\%. Em três casos o gás foi colocado no segundo dia pósdeslocamento, o quarto paciente no $15^{\circ}$ dia, o quinto com 40 dias e o sexto com 60 dias de pós-operatório.

Com uma seringa de $10 \mathrm{ml}$ conectada em uma agulha de insulina $(13 \times 4,5)$ era aspirado $2,0 \mathrm{ml}$ do gás $\mathrm{C} 3 \mathrm{~F} 8$ do recipiente, logo em seguida expulsava-se $0,4 \mathrm{ml}$ para atingir a quantidade desejada $(1,6 \mathrm{ml})$ para então completar a seringa com ar ambiente até $10 \mathrm{ml}$ e ficar, ao final, com uma concentração próxima de $16,0 \%$.

Dos cinco casos que foram realizados facoemulsificação, em dois utilizamos a mesma incisão superior temporal para introdução da cânula com o gás. Uma outra opção utilizada foi a punção corneana, justa-limbar com agulha de insulina (13 x $4,5)$ na região oposta ao descolamento, aspirava-se $0,1 \mathrm{ml}$ de humor aquoso da câmara anterior e injetava-se o gás. No caso da extração extra-capsular do cristalino, entramos superiormente às 12:00 horas com cânula adequada.

Imediatamente à colocação do gás $(0,5 \mathrm{ml}$ em câmara anterior) o paciente era avaliado em lâmpada de fenda para verifi- car a localização justa-estromal da membrana de Descemet/ endotélio, além de medir da pressão intra-ocular.

Os pacientes com descolamento superior e central da membrana eram orientados a ficar em posição de decúbito elevado por dois dias e no caso do descolamento inferior solicitamos decúbito dorsal com leve supraversão. Essas posições são importantes para maior contato da bolha com o local do descolamento.

Em um caso houve aumento da pressão intra-ocular, sendo utilizado acetazolamida $250 \mathrm{mg}$ 6/6 horas até controle adequado.

No pós-operatório de todos os pacientes foi prescrito tobramicina com dexametasona de $6 / 6$ horas por 10 dias.

Dois dias após, os pacientes eram reavaliados para observação do edema, acuidade visual, medida da pressão intraocular e posição da membrana da Descemet. Tivemos dificuldade em avaliar a AV no segundo dia por presença da bolha em eixo visual, mas no sexto dia já era possível medir a visão de todos os pacientes.

Os dados foram digitados em planilha do EXCEL, sendo as variáveis quantitativas contínuas avaliadas pelo teste $\mathrm{t}$ de Student.

\section{RESULTADOS}

Todos os pacientes tiveram sucesso com a colocação da bolha do gás C3F8. O edema corneano regrediu a partir do segundo dia com resolução completa até o quarto dia.

A média de idade dos pacientes foi de 71,3 \pm 9,3 anos, sendo quatro do sexo feminino e dois do sexo masculino. A acuidade visual com correção no sexto dia de pós-operatório foi de 0,40 (20/50) em quatro pacientes, um ficou com 0,25 (20/80) e outro com 0,20 (20/100). Não houve diferença estatisticamente siguinificativa entre o PAM e acuidade visual com 45 dias de pós-operatório ( $\mathrm{p}=0,0722$ ). Com 45 dias após introdução da bolha a AV melhorou significantemente em relação ao pré-operatório (Tabela 1).

Como mostra a tabela 1 , cinco casos tinham descolamento da Descemet superior e central. Em um notamos descolamento atingindo a região central até inferior com contato Descemet/ estroma apenas medial periférico, com rasgo central da membrana e envelopamento das abas (secundário provavelmente ao edema feito no final da facoemulsificação pela entrada de serviço). Nesse caso, em especial, entramos com dupla via, irrigamos até desdobrar as abas para então colocarmos o gás.

Durante o acompanhamento dos seis pacientes (mínimo de seis meses) não notamos novo descolamento da membrana de Descemet, com manutenção da acuidade visual.

A pressão intra-ocular de um olho aumentou oito horas após a colocação da bolha (45 mmHg às 11:00 horas), melhorando com uso de acetazolamida. Os demais olhos, não apresentaram picos da pressão intra-ocular havendo uma variação entre $14 \mathrm{mmHg}$ e $18 \mathrm{mmHg}$ no segundo dia de pós-operatório.

Nas figuras (Figuras 1 a 6) acompanhamos alguns casos de descolamentos da membrana de Descemet até clareamento corneano pós-bolha. 


\begin{tabular}{|c|c|c|c|c|c|c|}
\hline Casos & $\begin{array}{l}\text { Idade } \\
\text { (anos) }\end{array}$ & $\begin{array}{l}\text { PAM pré- } \\
\text { facectomia }\end{array}$ & $\begin{array}{l}\text { Localização do } \\
\text { descolamento }\end{array}$ & $\begin{array}{c}\text { AV } \\
\text { pré-operatória }\end{array}$ & $\begin{array}{l}\text { AV c/c } \\
45 \text { DPO }\end{array}$ & $\begin{array}{c}\text { Tipo de } \\
\text { facectomia }\end{array}$ \\
\hline 1 & 83 & 0,20 & Superior e central & Conta dedos a 2 metros & 0,25 & Extracapsular \\
\hline 2 & 72 & 0,67 & Superior e central & Conta dedos a 4 metros & 0,80 & Facoemulsificação \\
\hline 3 & 68 & 0,50 & Superior e central & Conta dedos a 4 metros & 0,50 & Facoemulsificação \\
\hline 4 & 81 & 0,29 & Superior e central & Conta dedos a 3 metros & 0,33 & Facoemulsificação \\
\hline 5 & 59 & 0,50 & Superior e central & Conta dedos a 2 metros & 0,67 & Facoemulsificação \\
\hline 6 & 65 & 0,80 & Superior, central e inferior & Conta dedos a 1 metro & 0,80 & Facoemulsificação \\
\hline
\end{tabular}

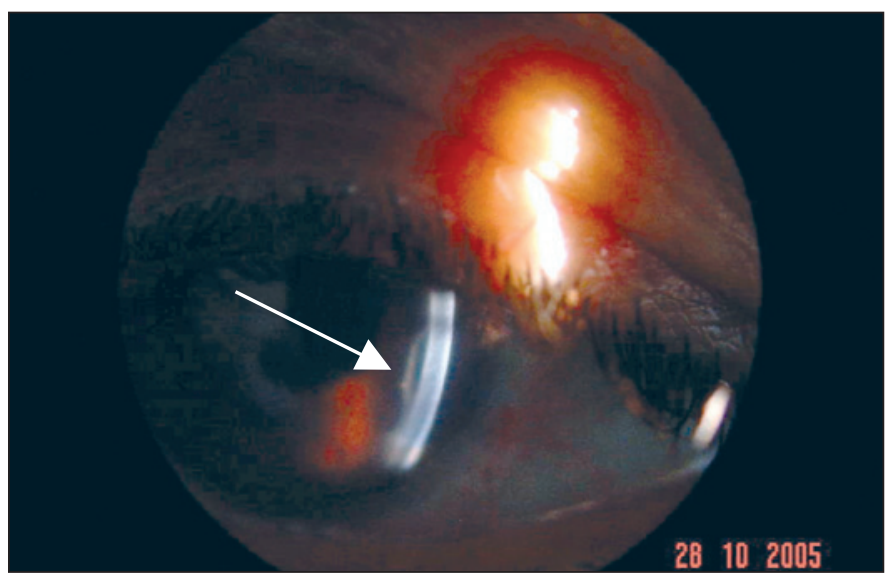

Figura 1 - Descolamento da Descemet (linha posterior)

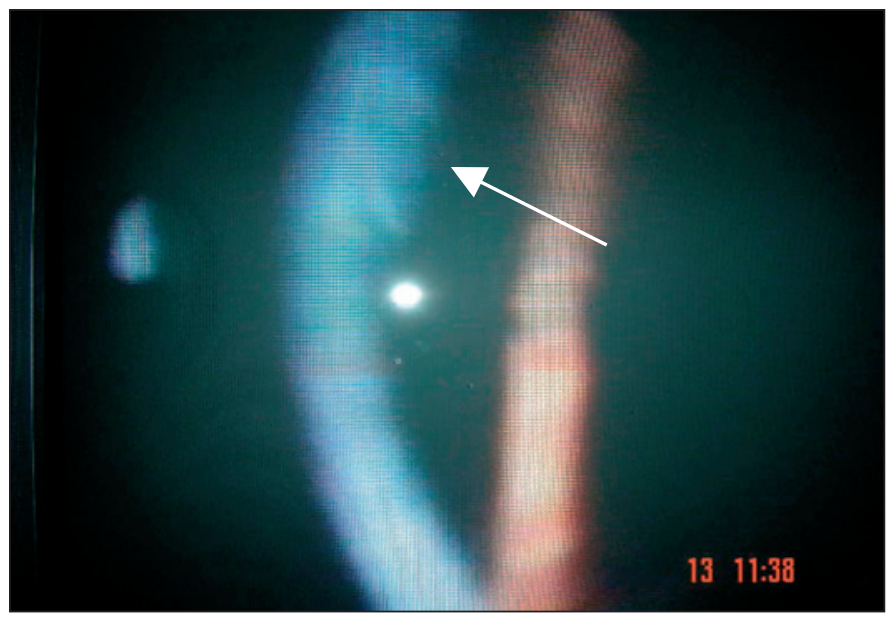

Figura 3 - Edema superior (pré-operatório)

\section{DISCUSS ÃO}

O descolamento da membrana de Descemet não é uma complicação freqüente no pós-operatório da cirurgia de catarata, mas causa uma baixa visual intensa quando atinge o eixo visual e, quando o edema persiste por muito tempo a ceratopatia bolhosa é uma ocorrência que incomoda e baixa a qualidade de vida dos pacientes.

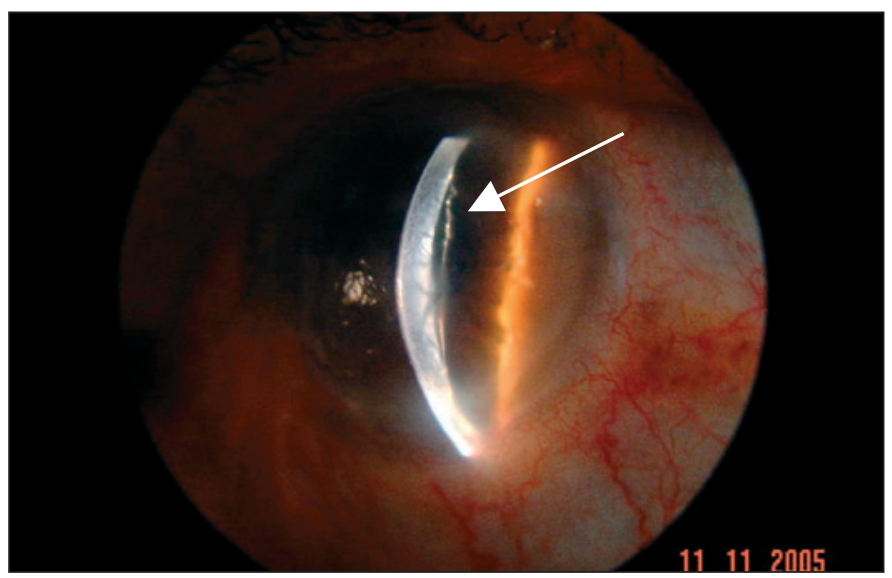

Figura 2 - Descolamento da Descemet (linha posterior)

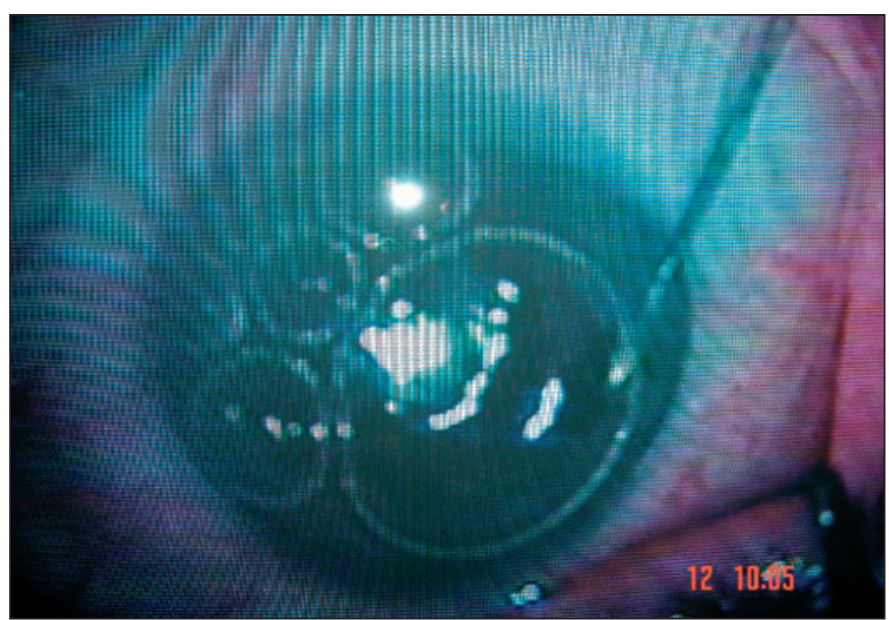

Figura 4 - Introdução da bolha C3F8 com agulha de insulina

O transplante de córnea é uma alternativa já conhecida para esses casos. A sutura da Descemet englobando a espessura total da córnea, a colocação de viscoelástico com bolha de ar ou a introdução de SF6 com ou sem sutura também são relatados na literatura ${ }^{(2,4,7-8)}$.

A colocação da bolha de ar não é efetiva, principalmente em descolamentos extensos, que atingem o eixo visual, já que a sua absorção é rápida. $\mathrm{O}$ uso de viscoelástico aumenta a pressão 


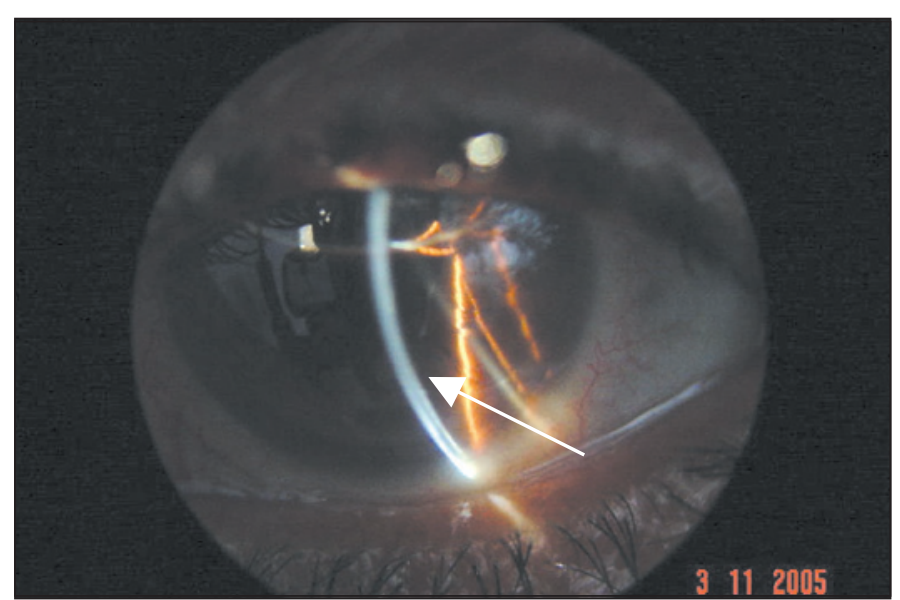

Figura 5 - Córnea sem edema no 10 dia de pós-operatório

intra-ocular, podendo também ficar resíduo entre a Descemet e o estroma posterior, bloqueando a adesão. A sutura da membrana de Descemet é traumática, de difícil realização, além de ter um alto índice de redescolamento. O transplante de córnea é uma opção radical que deve ser deixado para último $\operatorname{caso}^{(9-10)}$.

Uma boa opção para reposicionamento da membrana de Descemet, em extensos descolamentos, é a introdução unicamente do gás C3F8 não expansivo, sob anestesia tópica. Apesar de ser um método conhecido, é citado pela primeira vez na literatura nacional. Há uma permanência maior do gás dentro da câmara anterior (sete a 14 dias) mantendo a membrana de Descemet por mais tempo colada ao estroma, contribuindo assim para a adesão definitiva ${ }^{(11-13)}$.

Logo após a colocação da bolha do gás a membrana de Descemet pode ser observada em lâmpada de fenda colada ao estroma. $\mathrm{O}$ reestabelecimento da acuidade visual é rápida e no sexto dia de pós-operatório todos os olhos apresentam visão útil.

Houve um caso de pico hipertensivo pós-C3F8. Pode ter havido uma leve expansão do gás com conseqüente aumento da pressão por aumento do volume da câmara anterior. Acreditamos nessa hipótese por não ter sido notado bloqueio angular nesse paciente.

No caso do descolamento inferior foi necessário o retorno do paciente ao bloco por aposicionamento da bolha sobre uma dobra da Descemet. A bolha foi então retirada, a dobra foi desfeita utilizando uma dupla via irrigada com ringer lactato para então introduzirmos uma nova bolha.

Em termos de custos, se a clínica já tem serviço de retina e possui o cilindro de $\mathrm{C} 3 \mathrm{~F} 8$, consideramos a quantidade de gás utilizada irrisória (1,6 ml em uma seringa de $10 \mathrm{ml}=\mathrm{R} \$ 1,21)$. Caso o serviço não possua o cilindro, o investimento inicial é de 1.520 reais em um cilindro de $125 \mathrm{~g}$ de C3F8 ou de 691 reais no cilindro de 20 g. Já que o descolamento de Descemet não é complicação freqüente, a utilização do gás com esse propósito será rara, podendo ser mais adequado, financeiramente, o encaminhamento para serviço de referência.

Um achado biomicroscópico descoberto nesse trabalho

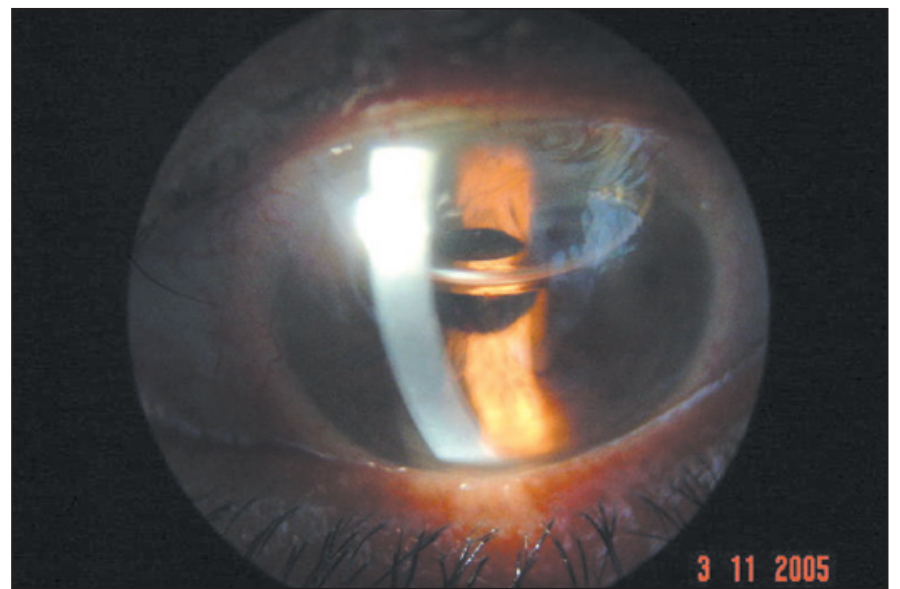

Figura 6 - Córnea clara no 10 dia de pós-operatório

foi a observação de estrias posteriores em estroma, mesmo com descolamento da Descemet na área das estrias, nos levando a concluir que as estrias notadas em pós-operatório de cirurgia de catarata ou outras cirurgias de segmento anterior, não são primariamente estrias de Descemet, como conhecida e repassada há décadas em escolas de oftalmologia, mas sim estrias do próprio estroma posterior.

\section{CONCLUSÃO}

Partindo-se do princípio que o descolamento da membrana de Descemet é complicação rara após facectomia, consideramos o número de olhos suficientes para demonstrar que o uso de C3F8 nesses casos é método seguro, efetivo e de fácil execução.

\section{AGRADECIMENTOS}

Dr. Levy da Rocha Lucena, Dra. Maria do Socorro Aguiar Ribeiro de Lucena e Dr. Abelardo Targino.

\section{ABSTRACT}

Introduction: Descemet's membrane detachment is a rare but serious complication following cataract surgery. Extensive detachments that affect the visual axis may result in poor vision and require penetrating corneal grafts. Some alternatives have been used to reattach Descemet's membrane: air bubble, transcorneal sutures and intracameral viscoelastic gel plus SF6 or C3F8 gas. Purpose: To describe the use of non-expanding $(16 \%)$ intracameral C3F8 to reattach Descemet's membrane, in six patients, following cataract surgery. Methods: The patient's mean age was $71.3 \pm 9.3$. Four of the patients were females and two masculine. Immediately afters the gas injection the patient was submitted to slit-lamp evaluation to verify the position of Descemet's membrane. The patients who had 
eyes with central or superior detachments were told to sit upright for two days. The patients whose eyes had inferior detachments were asked to lie on their back. Results: All patients had successful reattachment of Descemet's membrane after the gas injection. We observed increase in intraocular pressure in one case. The corneal edema regressed after the second day with total resolution by the fourth day. Visual acuity improved in all cases after regression of the edema and remained stable after forty-five days. Conclusion: The use of non-expanding C3F8 alone for reattaching Descemet's membrane is reported for the first time in the Brazilian literature. Improvement in visual acuity is fast, the method is safe, effective and easily performed under topical anesthesia.

Keywords: Cataract extraction/adverse effects; Descemet's membrane; Fluorocarbons/administration \& dosage; Corneal edema/drug therapy; Injections

\section{REFERÊNCIAS}

1. El Ammar Muller A, Meneghi RM de, Ferreira RLP, Putz C. Córnea, membrana de Descemet. In: Putz C, editor. Oftalmologia ciências básicas. Rio de Janeiro: Cultura Medica; 2001. p.124.

2. Nouri M, Pineda R Jr, Azar D. Descemet membrane tear after cataract surgery. Semin Ophthalmol. 2002;17(3-4):115-9.
3. Kim T, Hasan SA. A new technique for repairing descemet membrane detachments using intracameral gas injection. Arch Ophthalmol. 2002;120(2):181-3.

4. Breihenbach K, Kohlhaas M, Schwartz, R. Detachment of the Descemet membrane as a complication after phacoemulsification. Means of surgical Reattachment [abstract]. In: 97 th DOG Annual Meeting, 1999. Meeting. University-Eye-Hospital. Hamburg. [cited 2005 Sep 15]. Available from: http:// www.dog.org/1999/e-abstract99/475.html

5. Mulhern M, Barry P, Condon P. A case of Descemet's membrane detachment during phacoemulsification surgery. Br J Ophthalmol. 1996;80(2):185-6. Comment in: Br J Ophthalmol. 1997;81(2):174.

6. Walland MJ, Stevens JD, Steele AD. Repair of Descemet's membrane detachment after intraocular surgery. J Cataract Refract Surg. 1995;21(3):250-3. Comment in: J Cataract Refract Surg. 1995;21(6):598-9.

7. Koh JW, Woo WJ, Na KS. A case of total Descemet's membrane detachment treated by mon-expansible SF6 gas infusion. J Korean Ophthalmol Soc. 2002; 43(12):2598-602.

8. Ocakoglu O, Ustundag C, Devranoglu K, Yildirim R, Ozkan S. Repair of Descemet's membrane detachment after viscocanalostomy. J Cataract Refract Surg. 2002;28(9):1703-6.

9. Gault JA, Raber IM. Repair of Descemet's membrane detachment with intracameral injection of $20 \%$ sulfur hexafluoride gas. Cornea. 1996;15(5):483-9.

10. Sparks GM. Descemetopexy. Surgical reattachment of stripped Descemet's membrane. Arch Ophthalmol. 19671;78(1):31-4.

11. Macsai MS, Gainer KM, Chisholm L. Repair of Descemet's membrane detachment with perfluoropropane (C3F8) Cornea. 1998;17(2):129-34. Comment in: Cornea. 1998;17(4):457.

12. Shah M, Bathia J, Kothari K. Repair of late Descemet's membrane detachment with perfluoropropane gas. J Cataract Refract Surg. 2003;29(6):1242-4.

13. Kremer I, Stiebel H, Yassur Y, Weinberger D. Sulfur hexafluoride injection for Descemet's membrane detachment in cataract surgery. J Cataract Refract Surg. 1997;23(10):1449-53. Comment in: J Cataract Refract Surg. 1999;25(2):160.

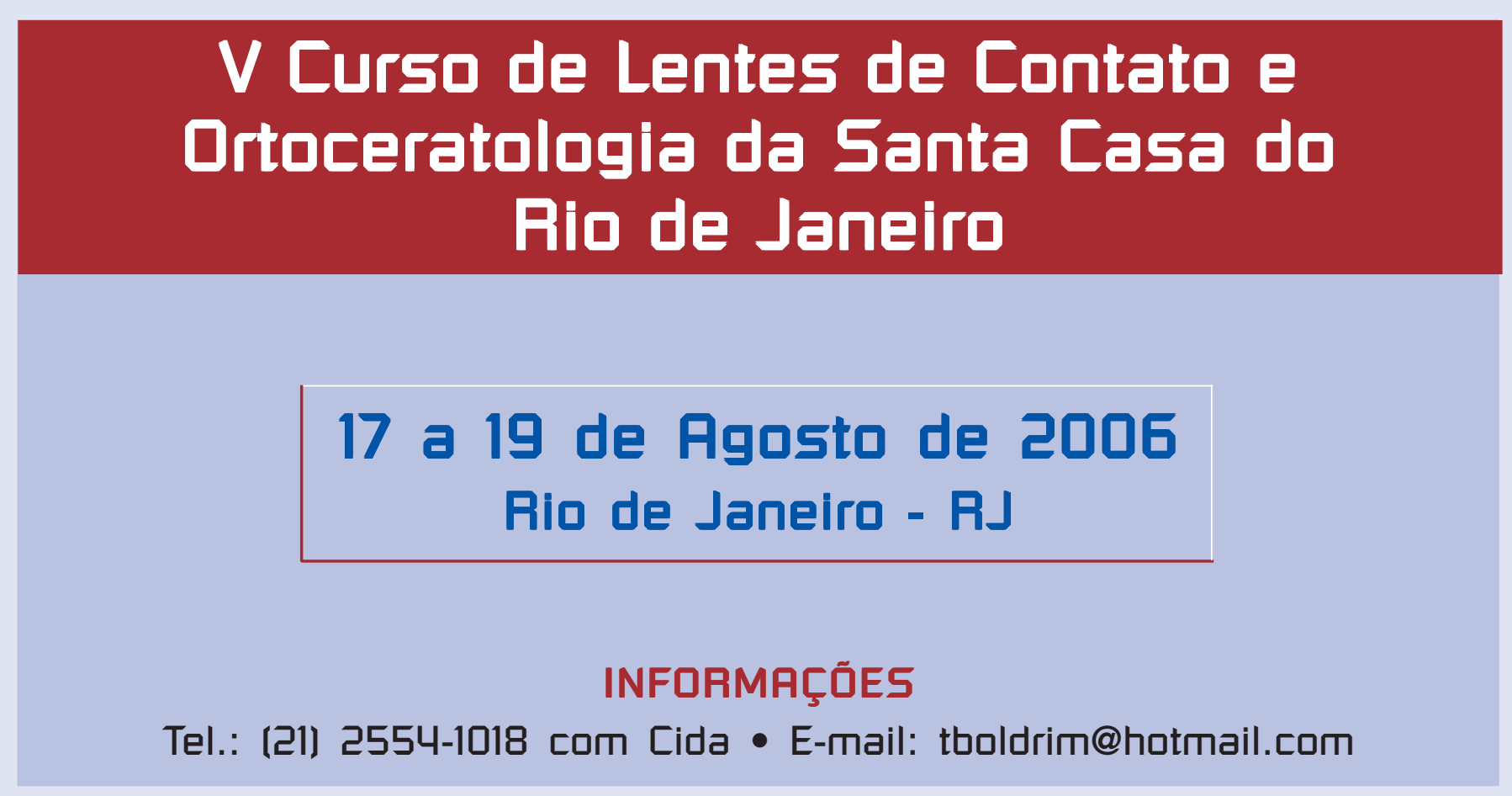

